\title{
Visualizing hyperbolic Voronoi diagrams
}

\author{
Frank Nielsen \\ Sony Computer Science Laboratories, Japan \\ Ecole Polytechnique, France \\ Frank.Nielsen@acm.org
}

\author{
Richard Nock \\ NICTA, Australia \\ CEREGMIA, UAG, France \\ rnock@martinique.univ-ag.fr
}

\begin{abstract}
We present an interactive software, HVD, that represents internally the $k$-order hyperbolic Voronoi diagram of a finite set of sites as an equivalent clipped power diagram. HVD allows users to interactively browse the hyperbolic Voronoi diagrams and renders simultaneously the diagram in the five standard models of hyperbolic geometry: Namely, the Poincaré disk, the Poincaré upper plane, the Klein disk, the Beltrami hemisphere and the Weierstrass hyperboloid.
\end{abstract}

\section{INTRODUCTION}

The Voronoi Diagram (VD) of a $d$-dimensional point set $\mathcal{P}=\left\{P_{1}, \ldots, P_{n}\right\}$ partitions the space $\mathbb{X}$ into proximity cells $V_{i}$ 's:

$$
V\left(P_{i}\right)=\left\{X \in \mathcal{X}: D\left(X, P_{i}\right) \leq D\left(X, P_{j}\right), \forall P_{j} \in \mathcal{P}\right\} .
$$

The ordinary $\mathrm{VD}$ is obtained for the Euclidean distance $D(P, Q)=\left\|p^{C}-q^{C}\right\|$, where $p^{C}$ and $q^{C}$ denote the Cartesian coordinates of $P$ and $Q$, respectively. The $k$-order VD generalizes the $\operatorname{VD}(k=1)$ by considering all the subsets of size $k, \mathcal{P}_{k}=\left(\begin{array}{l}\mathcal{P} \\ k\end{array}\right)=\left\{\mathcal{K}_{1}, \ldots, \mathcal{K}_{l}\right\}$ with $l=\left(\begin{array}{l}n \\ k\end{array}\right)$, and partitioning $\mathbb{X}$ with the non-empty cells:

$V_{k}(\mathcal{K})=\{X: \forall Q \in \mathcal{K}, \forall R \in \mathcal{P} \backslash \mathcal{K}, D(X, Q) \leq D(X, R)\}$.

In practice, the ordinary $k$-order VDs can be computed efficiently, without enumerating all the $k$-subsets of $\mathcal{P}$, by calculating the $k$-level of an arrangement of $n$ hyperplanes in dimension $d+1$ (see [3], §13.3, pp. 315-326). In machine learning, $k$-order VDs are used for studying the $k$-nearest neighbor classification rules.

The Hyperbolic geometry [2] is a non-Euclidean geometry where the Euclidean parallel fifth postulate is replaced by the property that there exists infinitely many lines passing through a given point and not intersecting another given line. Computing the hyperbolic Voronoi diagrams has been studied in $[4,1]$ and find increasingly many applications in graph drawing and visualization, meshing (via the Poincaré Permission to make digital or hard copies of part or all of this work for personal or classroom use is granted without fee provided that copies are not made or distributed for profit or commercial advantage and that copies bear this notice and the full citation on the first page. Copyrights for thirdparty components of this work must be honored. For all other uses, contact the Owner/Author.

Copyright is held by the owner/author(s).

SoCG'14, June 8-11, 2014, Kyoto, Japan. ACM 978-1-4503-2594-3/14/06.

http://dx.doi.org/10.1145/2582112.2595647

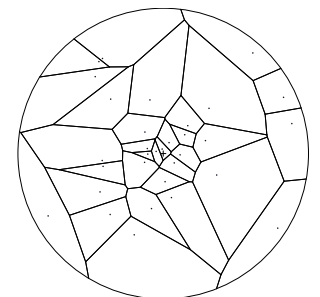

(a)

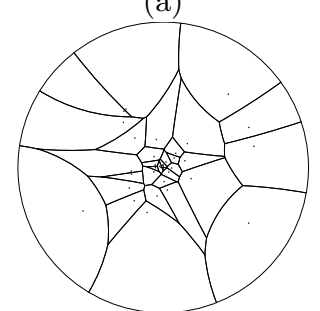

(c)

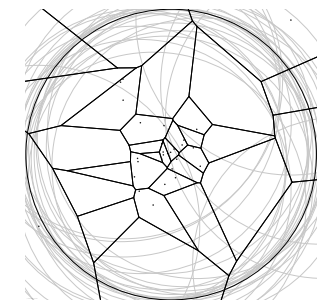

(b)

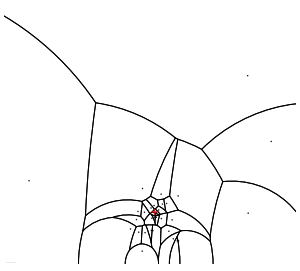

(d)
Figure 1: Klein hyperbolic Voronoi diagram (a) is equivalent to a clipped power diagram (b). The HVD viewed in the Poincaré (c) and in the upper plane (d) models. Zoom in those vector graphics.

uniformization theorem), network routing, machine learning, information geometry, percolation, etc.

\section{KLEIN HYPERBOLIC VD}

The Klein disk model of hyperbolic geometry $\mathbb{H}^{d}$ considers the open unit disk $\mathbb{B}_{1}$, and defines for two points $P$ and $Q$ of $\mathbb{H}^{d}$ with Cartesian coordinates $p^{K}$ and $q^{K}$ in $\mathbb{B}_{1}$ the hyperbolic distance:

$$
\begin{aligned}
D(P, Q) & =d_{K}\left(p^{K}, q^{K}\right), \\
& =\operatorname{arcosh}\left(\frac{1-\left\langle p^{K} \mid q^{K}\right\rangle}{\sqrt{\left(1-\left\langle p^{K} \mid p^{K}\right\rangle\right)\left(1-\left\langle q^{K} \mid q^{K}\right\rangle\right)}}\right),
\end{aligned}
$$

where $\operatorname{arcosh}(x)=\log \left(x+\sqrt{x^{2}-1}\right)$ and $\langle p \mid q\rangle=\sum_{i=1}^{d} p_{i} q_{i}$ denotes the (euclidean) inner product. Klein bisectors are hyperplanes [4] and therefore the $k$-order hyperbolic VDs can be computed as an equivalent power diagram (PD) of a corresponding set of weighted points restricted to $\mathbb{B}_{1}$. Technically, we may assume w.l.o.g. that weights are non-negative and set the minimum weight to zero. Indeed, the power bisector of $\left(P_{i}, w_{i}\right)$ and $\left(P_{j}, w_{j}\right)$ is identical to the power bisector of $\left(P_{i}, w_{i}+\Delta\right)$ and $\left(P_{j}, w_{j}+\Delta\right)$ for any $\Delta \in \mathbb{R}$. Figure 1 depicts the hyperpolic VD calculated internally as 


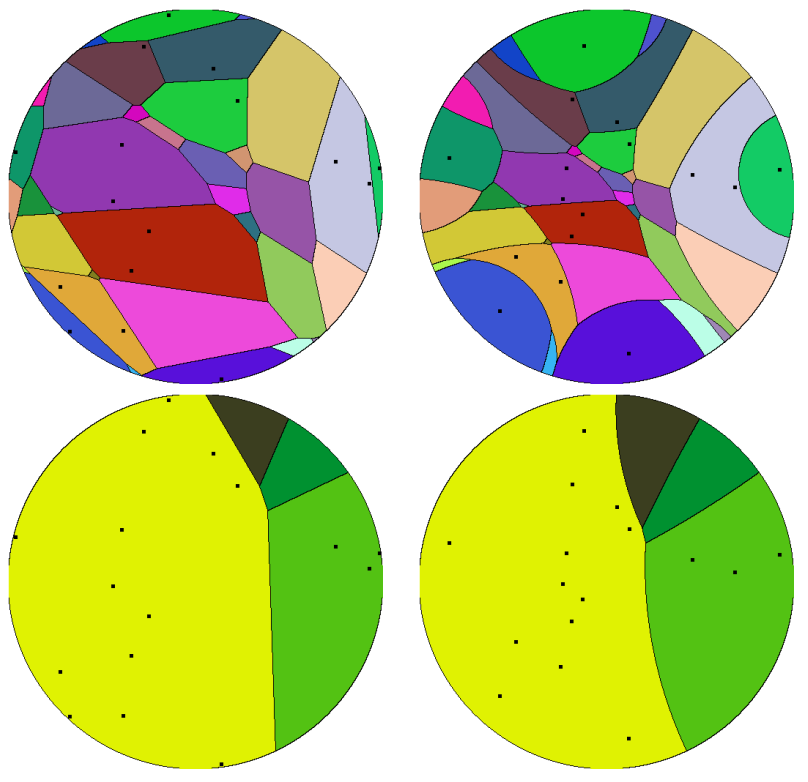

Figure 2: $k$-order HVDs in the Klein and Poincaré disk models for $n=16$ sites and $k=3$ (top) and $k=15$ (bottom). The left column shows the Klein affine HVDs and the right column displays the equivalent Poincaré HVDs in the conformal disk model. The $(k-1)$-order diagram, called the farthest HVD, can be used to characterize the circumcenter of the smallest enclosing hyperbolic disk.

a $\mathrm{PD}$ and rendered either in the Klein disk model $(\mathrm{K})$, the Poincaré disk model $(\mathrm{P})$ or the Poincaré upper space model (U). Figure 2 shows the $k$-order hyperbolic VD in the disk models. Since bisectors and geodesics are affine in the Klein model, many standard computational geometric gems can be used. However, for visualization purposes, the conformal Poincaré disk and upper space models are often preferred since they do not distort angles. Note that Poincaré and Klein bisectors meet at the bounding sphere. In 2D, browsing the HVDs is performed using Möbius transformations [4] preserving the unit disk (isometries).

The video illustrates the HVDs interactively by adding, moving, or animating generators with motions, and choosing the rendering modes ( $\mathrm{K}, \mathrm{P}$ or $\mathrm{U}$, and overlaying $\mathrm{KP})$.

\section{FIVE STANDARD MODELS}

By adding an extra dimension, we may simultaneously visualize the K, P and U models [2]. Furthermore, we add the Beltrami hemisphere (B) and the Weierstrass hyperboloid model (upper sheet, W). Note that the hyperbolic distance is an intrinsic quantity independent of the chosen model, and can therefore be expressed equivalently:

$$
\begin{aligned}
D(P, Q) & =d_{K}\left(p^{K}, q^{K}\right)=d_{P}\left(p^{P}, q^{P}\right)=d_{U}\left(p^{U}, q^{U}\right), \\
& =d_{W}\left(p^{W}, q^{W}\right)=d_{B}\left(p^{B}, q^{B}\right) .
\end{aligned}
$$

The video shows how those models relate to each others (see Figure 3), and allows one to inspect the HVDs in these five standard models.

\section{SOFTWARE}

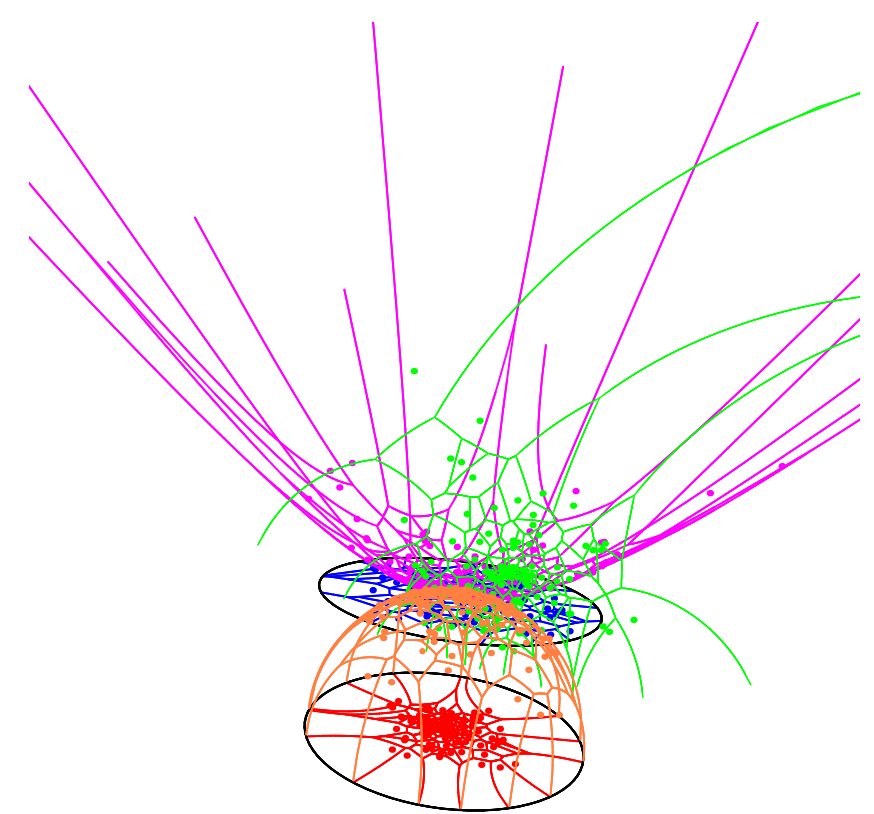

Figure 3: Visualizing the standard models of $\mathbb{H}^{d}$ in $\mathbb{R}^{d+1}$ : Poincaré disk $(\mathbf{P}$, red $)$, Beltrami hemisphere (B, orange), Klein disk (K, blue), Poincaré upper space (U, green) and Weierstrass hyperboloic (W, purple). You can zoom in this vector graphics.

HVD has been implemented in the Processing language (version 2.1.1, processing.org). The program relies on a Java $^{\mathrm{TM}}$ in-house library, called HGL (standing for Hyperbolic Geometry Library), for manipulating geometric entities such as points, balls, geodesics, bisectors, transformations, etc. in real hyperbolic spaces of arbitrary dimension $d$ and constant negative curvature $\kappa<0$. Klein model is wellsuited to computation since both geodesics and bisectors are affine and there is no particular degeneracies to consider. Indeed, in the Poincaré $\mathrm{P}$ and $\mathrm{U}$ models, the spherical bisectors may degenerate to affine hyperplanes (sphere radius tending to infinity), and therefore be numerically unstable. HVD allows to export various renderings in both SVG and PDF vector graphics formats (e.g., zoom in Figure 1). HVD also implements other models of hyperbolic geometry and supports other operations like the convex hull or smallest enclosing ball, etc.

\section{REFERENCES}

[1] M. Bogdanov, O. Devillers, and M. Teillaud. Hyperbolic Delaunay complexes and Voronoi diagrams made practical. In Proc. SoCG, pages 67-76, 2013.

[2] J. W. Cannon, W. J. Floyd, R. Kenyon, and W. R. Parry. Hyperbolic geometry. In Flavors of Geometry, pages 59-115. Cambridge University Press, 1997.

[3] H. Edelsbrunner. Algorithms in Combinatorial Geometry. Springer-Verlag New York, Inc., 1987.

[4] F. Nielsen and R. Nock. Hyperbolic Voronoi diagrams made easy. In International Conference on Computational Science and Its Applications (ICCSA), pages $74-80,2010$. See also arxiv 1210.8234 . 